\title{
Acute Kidney Injury in Patients with Liver Cirrhosis: Prevalence, Predictors, and In-Hospital Mortality at a District Hospital in Ghana
}

\author{
Amoako Duah $\mathbb{D}^{1},{ }^{1}$ Francisca Duah $\mathbb{D},{ }^{2}$ Daniel Ampofo-Boobi $\mathbb{D},{ }^{3}$ Bright Peprah Addo $\mathbb{D},{ }^{4}$ \\ Foster Osei-Poku $\mathbb{D}^{3},{ }^{3}$ and Adwoa Agyei-Nkansah $\mathbb{1}^{5}$ \\ ${ }^{1}$ Department of Medicine, University of Ghana Medical Centre, Accra, Ghana \\ ${ }^{2}$ Laboratory Department, Ga-North Municipal Hospital, Ofankor, Ghana \\ ${ }^{3}$ Department of Medicine, St. Dominic Hospital, Akwatia, Ghana \\ ${ }^{4}$ Department of Biostatistics, St. Dominic Hospital, Akwatia, Ghana \\ ${ }^{5}$ Department of Medicine, University of Ghana Medical School, Korle-Bu Teaching Hospital, Accra, Ghana
}

Correspondence should be addressed to Amoako Duah; amoakoduah@yahoo.com

Received 14 May 2021; Revised 30 November 2021; Accepted 11 February 2022; Published 21 February 2022

Academic Editor: Maria Irene Bellini

Copyright (C) 2022 Amoako Duah et al. This is an open access article distributed under the Creative Commons Attribution License, which permits unrestricted use, distribution, and reproduction in any medium, provided the original work is properly cited.

Background. Acute kidney injury (AKI) is one of the most severe complications of cirrhosis and portends an ominous prognosis with an estimated mortality of about $50 \%$ in a month and $65 \%$ within a year. Infection and hypovolemia have been found to be the main precipitating factors of AKI in liver cirrhosis. Early detection and treatment of AKI may improve outcomes. AKI in patients with liver cirrhosis in Ghana and their impact on inpatient mortality are largely unknown. This study was aimed at determining the prevalence, precipitating factors, predictors, and in-hospital mortality of AKI in patients with liver cirrhosis admitted to a district hospital in Ghana. Methods. Consecutive hospitalized patients with liver cirrhosis from 1 January 2018 to 30 April 2020 were recruited. Patient's demographic data and clinical features were collected using a standardized questionnaire. Biochemical and haematological tests as well as abdominal ultrasound scans were done for all patients. All patients were then followed up until discharge or death. Results. There were 117 (65.4\%) males out of the 179 patients with a mean age of 49.94 and 45.84 years for those with and without AKI, respectively. The prevalence of AKI was 27.9\% (50/179). Out of 50 participants with AKI, $64.0 \%(32 / 50)$ died, contributing $41.0 \%$ of all in-patient mortality amongst participants. There was a significant association between AKI and death $(p \leq 0.001)$. The major precipitating factors of AKI were infections $(60.0 \%)$, hypovolemia $(20.0 \%)$ due to gastrointestinal bleeding and gastroenteritis, and refractory ascites (16.0\%). Alkaline phosphatase, INR, model for end-stage liver disease sodium, sodium, and blood urea nitrogen were independent predictors of AKI. Conclusion. AKI was common among patients with liver cirrhosis with high in-patient mortality. Identification of these precipitants and independent predictors of AKI may lead to prompt and targeted treatment with reduction in patient mortality.

\section{Introduction}

Liver cirrhosis is a worrying medical condition globally, particularly in Ghana where hepatitis B and alcohol are important risk factors [1]. It is a sequel of all chronic liver diseases characterized by replacement of liver tissue by fibrosis, scar tissue, and regenerative nodules, resulting in increased loss of liver function [2]. Cirrhosis can remain compensated for many years before the development of a decompensating events. Decompensated cirrhosis is marked by the development of any of the following complications: jaundice, variceal hemorrhage, ascites, and encephalopathy. One of the deadly and common complications of liver cirrhosis, especially in decompensated cirrhosis, is AKI [3]. In patients with liver cirrhosis admitted to the hospital, $20 \%$ to $50 \%$ of them are diagnosed with acute kidney failure, while chronic kidney disease is present in approximately $1 \%$ [4-6]. AKI is one of the key predictors for short-term mortality in patients 
TABLE 1: Demographic characteristics and clinical features of cirrhotic patients with and without AKI.

\begin{tabular}{|c|c|c|c|}
\hline Demography & AKI, present $(N=50)$ & AKI, absent $(N=129)$ & $p$ value \\
\hline Age of patient (years, IQR) & $49.94(36.47,63.47)$ & $45.84(33.05,58.63)$ & 0.6 \\
\hline Sex of patient & & & 0.38 \\
\hline Male & $30(25.6)$ & $87(74.4)$ & \\
\hline Female & $20(32.3)$ & $42(67.7)$ & \\
\hline \multicolumn{4}{|l|}{ Blood pressure ( $m m H g, I Q R)$} \\
\hline Systolic BP & $106(92.15,119.85)$ & $108.7(93.8,123.56)$ & 0.268 \\
\hline Diastolic BP & $68.52(58.52,78.52)$ & $69.15(58.86,79.4)$ & 0.713 \\
\hline Length of stay (days) & $10.06(2.69,17.43)$ & $10.67(3.97,17.37)$ & 0.578 \\
\hline \multicolumn{4}{|l|}{ Outcome of admission } \\
\hline Discharge & $18(17.8)$ & $83(82.2)$ & \\
\hline Death & $32(41.0)$ & $46(59.0)$ & 0.001 \\
\hline \multicolumn{4}{|l|}{ Clinical symptoms } \\
\hline Ascites & & & 0.201 \\
\hline Mild & $1(16.7)$ & $35(83)$ & \\
\hline Moderate & $20(38.5)$ & $32(61.5)$ & \\
\hline Severe & $23(25.6)$ & $67(74.4)$ & \\
\hline No ascites & $6(19.4)$ & $25(80.6)$ & \\
\hline Fever & $12(37.5)$ & $20(62.5)$ & 0.183 \\
\hline Infections & $30(42.8)$ & $40(57.2)$ & 0.038 \\
\hline Pedal oedema & $27(27.8)$ & $70(72.2)$ & 0.975 \\
\hline Jaundice & $28(35.9)$ & $50(64.1)$ & 0.058 \\
\hline Chills & $9(45)$ & $11(55)$ & 0.071 \\
\hline Abdominal pain & $18(28.1)$ & $46(71.9)$ & 0.966 \\
\hline Weight loss & $39(27.3)$ & $104(72.7)$ & 0.695 \\
\hline Encephalopathy & $27(47.4)$ & $30(52.6)$ & $<0.001$ \\
\hline UGI bleeding & $6(25)$ & $18(75)$ & 0.731 \\
\hline Others & $45(29.6)$ & $107(70.4)$ & 0.237 \\
\hline
\end{tabular}

UGI: upper gastrointestinal; BP: blood pressure.

with liver cirrhosis with mortality rates as high as $90 \%[7,8]$. There is a remarkable increase in length of hospital stay with high financial burden on patients with AKI [9]. In decompensated liver cirrhosis patients, Child-Pugh score and its components, which have been generally used to assess the severity of hepatic dysfunction together with AKI, have been found to be strong predictors of death [3].

Formerly, AKI in cirrhosis was defined as a $50 \%$ increase in serum creatinine (SCr) with a final value above $133 \mu \mathrm{mol} / 1$ [10]. Due to the high incidence of malnutrition and sarcopenia in patients with liver cirrhosis [11], this means milder degrees of AKI are not being diagnosed and therefore not given immediate attention and care. AKI is now defined as a rise in serum creatinine (SCr) of $\geq 50 \%$ from baseline or a rise in SCr by $\geq 26.5 \mu \mathrm{mol} / 1$ in $<48 \mathrm{~h}$ or equal to 1.5 times baseline, which has occurred within the prior 7 days [12, 13]. Hepatorenal syndrome (HRS) type 1 is a unique type of AKI.

Aetiology of AKI can be categorized into three main types. These are prerenal (results from decreased renal perfusion), intrinsic renal (acute tubular necrosis), and postrenal AKI. In patients on admission, acute tubular necrosis is a common type of intrinsic AKI. Prolonged hypotension leading to ischemia or nephrotoxic agents that are toxic to the tubular cells are the major causes of acute tubular necrosis. Enlargement of the prostate gland causing obstruction of the urinary tract is the common cause of postrenal AKI [2, 14]. The development of portal hypertension in patients with liver cirrhosis leading to a reduction in systemic vascular resistance as a result of primary arterial vasodilation in the splanchnic circulation is the primary cause of AKI in cirrhotics [15]. This mechanism primarily leads to hepatorenal syndrome. In addition, the underlying causes of liver cirrhosis can be the cause of intrinsic renal disease. These forms of nephropathy include glomerulonephritis associated with alcoholic cirrhosis and infections such as chronic hepatitis $\mathrm{B}$ and $\mathrm{C}$ and schistosomiasis [16]. Bacterial infections and hypovolemia resulting from gastrointestinal bleeding and over diuresis are other common causes of AKI [17].

Early identification and management of AKI may improve outcomes [18]. AKI in patients with liver cirrhosis in Ghana and their impact on inpatient mortality are largely unknown. This study was aimed at determining the prevalence, precipitating factors, predictors, and in-hospital mortality of AKI in patients with liver cirrhosis admitted at St. Dominic Hospital, Akwatia in Ghana. 
TABLE 2: Clinical characteristics of cirrhotic patients with AKI associated with and without ACLF.

\begin{tabular}{|c|c|c|c|}
\hline Variable & $\begin{array}{l}\text { AKI with ACLF } \\
\qquad(\mathrm{N}=16)\end{array}$ & $\begin{array}{l}\text { AKI without } \\
\operatorname{ACLF}(\mathrm{N}=34)\end{array}$ & $\begin{array}{c}p \\
\text { value }\end{array}$ \\
\hline Causes & & & 0.415 \\
\hline Alcohol & $10(62.4)$ & $14(41.2)$ & \\
\hline Alcohol/HBV & $0(0)$ & $1(2.9)$ & \\
\hline Alcohol/HBV/HCV & $1(6.3)$ & $0(0)$ & \\
\hline $\mathrm{HBV}$ & $4(25.0)$ & $13(38.2)$ & \\
\hline $\mathrm{HCV}$ & $1(6.3)$ & $2(5.9)$ & \\
\hline NAFLD & $0(0)$ & $1(2.9)$ & \\
\hline Unknown & $0(0)$ & $3(8.8)$ & \\
\hline Precipitating factors & & & 0.012 \\
\hline Ascites & $0(0)$ & $8(23.5)$ & \\
\hline Gastroenteritis & $0(0)$ & $3(8.8)$ & \\
\hline Infection & $13(81.3)$ & $19(55.8)$ & \\
\hline NIL & $0(0)$ & $2(5.9)$ & \\
\hline UGIB & $3(18.7)$ & $2(5.9)$ & \\
\hline Outcome & & & 0.001 \\
\hline Dead & $16(100 \%)$ & $16(47.1)$ & \\
\hline Live & $0(0 \%)$ & $18(52.9)$ & \\
\hline Sex of patient & & & 0.71 \\
\hline Female & $7(43.8)$ & $13(38.2)$ & \\
\hline Male & $9(56.2)$ & $21(61.8)$ & \\
\hline $\begin{array}{l}\text { Duration of stay } \\
\text { (days) }\end{array}$ & & & 0.305 \\
\hline $\begin{array}{l}\text { Median (minimum, } \\
\text { maximum) }\end{array}$ & $6(2,11)$ & $11.9(2,44)$ & \\
\hline \multicolumn{4}{|c|}{$\begin{array}{l}\text { HBV: hepatitis B virus; HCV: hepatitis C virus; NAFLD: nonalcoholic fatty } \\
\text { liver disease. }\end{array}$} \\
\hline \multicolumn{4}{|c|}{ TABLE 3: Child-Pugh classification. } \\
\hline Child-Pugh class & Frequency & $\mathrm{Pe}$ & rcentage \\
\hline Class A & 16 & & 8.9 \\
\hline Class B & 47 & & 26.3 \\
\hline Class C & 116 & & 64.8 \\
\hline Total & 179 & & 100.0 \\
\hline
\end{tabular}

\section{Methods}

2.1. Study Design, Site, and Participants. The research design was a prospective hospital-based study, conducted at the internal medicine department, St. Dominic hospital (SDH), Akwatia, from 1 January 2018 to 30th April 2020. The $\mathrm{SDH}$ is a 339-bedded mission hospital that serves Denkyembour district in the Eastern region of Ghana and serves as the main referral center for other district hospitals within the eastern region. The hospital provides various medical and surgical services including gastroenterology, neurology, general internal medicine, obstetrics and gynecology, child health, ophthalmology, general surgery, and endoscopy.

Liver transplantation services are not currently available at the study site and in Ghana as a whole. There is lack of
TABLE 4: Causes of liver cirrhosis.

\begin{tabular}{lcc}
\hline Causes & Frequency & Percent \\
\hline Alcohol & 72 & 40.2 \\
HBV & 65 & 36.3 \\
HCV & 6 & 3.4 \\
NAFLDX & 5 & 2.8 \\
Schistosomiasis & 4 & 2.2 \\
Alcohol/HBV/HCV & 1 & 0.6 \\
HBV/alcohol & 8 & 4.5 \\
HBV/HCV & 2 & 1.1 \\
HCV/alcohol & 2 & 1.1 \\
Alcohol/HBV/HCV & 1 & 0.6 \\
Unknown & 13 & 7.3 \\
Total & 179 & 100 \\
\hline HBV: hepatitis B virus; HCV: hepatitis C virus; NAFLD: nonalcoholic fatty \\
liver disease.
\end{tabular}

local clinical expertise and inadequate infrastructure to support liver transplant in Ghana. Appropriate legal framework of organ transplantation and guidelines are also not available in Ghana. Presently, patients requiring liver transplants and can afford travel to foreign countries where the services are available to have it done.

Overall, one hundred and seventy-nine (179) patients with liver cirrhosis admitted to the medical wards of SDH were recruited over the study period. The sample size was determined using the Cochran formula for sample size calculation, with an estimated prevalence of $12.9 \%$ for liver cirrhosis worldwide [3] and a $Z$-score of $95 \%$ confidence interval level (1.96) and a level of significance of 0.05 . The minimum sample size was calculated to be 171 . Due to the high attrition rate for prospective studies, 179 participants were consecutively recruited after meeting study criteria and giving informed consent.

\subsubsection{Inclusion Criteria}

(1) All adult patients above 18 years with diagnosis of liver cirrhosis

(2) Patients who provided informed consent

\subsubsection{Exclusion criteria}

(1) Patients with known preexisting chronic kidney disease

(2) Patients who had undergone renal transplantation

(3) Refusal to give informed consent

Diagnosis of liver cirrhosis was based on the presence of two or all three of the following [19]:

(1) Clinical signs of chronic liver disease (clubbing, palmar erythema, spider naevi, gynaecomastia, distended abdominal veins, female pubic hair pattern, encephalopathy, splenomegaly, or ascites) 
TABle 5: Precipitating factors for all AKI.

\begin{tabular}{lcc}
\hline & Frequency & Percent \\
\hline Infection & 30 & 60.0 \\
Refractory ascites & 8 & 16.0 \\
Hypovolemia & & \\
$\quad$ UGIB & 5 & 10.0 \\
$\quad$ Gastroenteritis & 5 & 10.0 \\
Unknown precipitant & 2 & 4.0 \\
Total & 50 & 100 \\
\hline
\end{tabular}

UGIB: upper gastrointestinal bleeding.

TABLE 6: Logistic regression analysis of AKI stage and its effect on in-hospital mortality.

\begin{tabular}{lcccccc}
\hline Outcomes & Odds ratio & SE & $p$ value & $\begin{array}{c}95 \% \text { conf } \\
\text { interval }\end{array}$ & Sig \\
\hline Stage 1a & 1 &. &. &. &. & \\
Stage 1b & 6 & 8.124 & .186 & .422 & 85.248 & \\
Stage 2 & 10.286 & 12.139 & .048 & 1.018 & 103.948 & $* *$ \\
Stage 3 & 48 & 63.119 & .003 & 3.647 & 631.76 & $* * *$ \\
Constant & .167 & .18 & .097 & .02 & 1.384 & $*$ \\
\hline
\end{tabular}

${ }^{* * *} p<.01,{ }^{* *} p<.05,{ }^{*} p<.1$.

(2) Impaired liver function consistent with cirrhosis (elevated INR and low serum albumin)

(3) Ultrasound diagnosis of cirrhosis (Shrunken or enlarged nodular liver with increased echotexture, blunt edge, and distorted architecture, with or without a dilated portal vein, thickened gallbladder wall, splenomegaly, or ascites)

AKI in patients with liver cirrhosis was defined by any one of the following: a rise in serum creatinine $(\mathrm{sCr})$ of $\geq 50 \%$ from baseline or a rise in $\mathrm{sCr}$ by $\geq 26.5 \mu \mathrm{mol} / \mathrm{l}$ in $<48 \mathrm{~h}$, or a rise in $\mathrm{sCr}$ to $\geq 1.5$ times baseline, occurring within the prior 7 days $[13,20]$. Baseline creatinine values were taken from the three-month preadmission periods. Admission values were used if they were normal or if prehospitalization baselines were not available. Those with no prehospitalization creatinine values, $\mathrm{sCr} \geq 133 \mathrm{umol} / \mathrm{l}$, were considered as having AKI at the time of admission. The staging of AKI in this study was adopted from a study published by $\mathrm{Xu}$ et al. [21]. AKI stage 1 was defined as a rise in $\mathrm{sCr} \geq 26.5 \mu \mathrm{mol} / \mathrm{l}$ or $\geq 1.5$ to 2 -fold from baseline, including AKI stages $1 \mathrm{~A}(\mathrm{sCr} \geq 133 \mu \mathrm{mol} / \mathrm{l})$ and $1 \mathrm{~B}(\mathrm{sCr} \geq 133 \mu \mathrm{mol} / \mathrm{l}$ ); AKI stage 2 was defined as a rise in $\mathrm{sCr} \geq 2$ to 3 -fold from baseline; AKI stage 3 was defined as a rise in $\mathrm{sCr} \geq 3$-fold from baseline or $\mathrm{sCr} \geq 353.6 \mu \mathrm{mol} / \mathrm{l}$ with an acute increase in $s \mathrm{Cr} \geq 26.5 \mu \mathrm{mol} / 1$ or initiation of renal replacement therapy. Initial AKI stage was defined as the stage of AKI first diagnosed during hospitalization. Progression of stage of AKI or no progression was determined by the last AKI stage close to discharge or death in relation to the initial AKI stage.
Patients with AKI associated with acute on chronic liver failure (ACLF) were differentiated from those with AKI and without ACLF.

In this study, ACLF was defined as acute worsening of preexisting liver cirrhosis (decompensated or compensated) usually related to a precipitating event and associated with short-term mortality due to multiple organ failure. Organ failure was defined using Chronic Liver Failure-Sequential Organ Failure (CLIF-SOFA) score which includes six types of organ failure: liver, renal, coagulation, cerebral, respiratory, and circulatory [22].

2.2. Data Collection. Appropriate questionnaires were administered to those who met the inclusion criteria and gave their consent to be part of the study after thoroughly explaining the study to them. The data collected included sociodemographic data (age, sex, occupation, etc.), clinical features (spider angioma, jaundice, abdominal pain, weight loss, confusion, hematemesis, palmar erythema, ascites, asterixis, hepatomegaly, splenomegaly, abdominal vein collaterals, etc.), and alcohol use. Initial data on laboratory and imaging findings were obtained within a day of admission. Ascites was graded as mild (detectable only on ultrasound), moderate (visible, moderate symmetrical abdominal distension), or severe (marked abdominal distension) [23]. Hepatic encephalopathy was graded from grade 0 to IV, as per the West Haven criteria [24]. Mean arterial pressure (MAP), partial pressure of arterial oxygen $(\mathrm{PaO} 2)$, and pulse oximetric saturation ( $\mathrm{SpO} 2)$ were obtained.

Risk factors/aetiological spectrums of liver cirrhosis were defined as follows:

Chronic HBV was defined as a positive HBsAg result

Chronic HCV was defined as a positive anti-HCV antibody result

Alcoholic aetiology was made when patient's declared alcohol consumption was more than 21 units of alcohol for men or 14 units for women per week when measurable or local alcohol beverage consumption was three times per week in the past five years and correlated with biological abnormalities related to alcohol consumption [25].

Hepatic schistosomiasis was defined as the presence of Schistosoma mansoni ova in stool and/or ultrasound findings of hepatic schistosomiasis features such as periportal fibrosis.

NAFLD aetiology was made when patients have risk factors for NAFLD and its progression and excluding other causes of liver cirrhosis.

Unidentified aetiology refers to CLD patients in whom no etiology was traced.

\subsection{Measurements}

2.3.1. Blood and Urine Tests. Ten (10) mls of blood was taken for hematological, biochemical, and serological tests. Hematological and biochemical workup included hemoglobin, total leukocyte count, platelet count, prothrombin time/INR, and serum concentrations of bilirubin (total and 
TABLE 7: Laboratory parameters of the study participants.

\begin{tabular}{lccc}
\hline Laboratory parameters & AKI, present $(N=50)$ & AKI, absent $(N=129)$ & $p$ value \\
\hline WBC $\left(10^{9} / \mathrm{l}\right)$ & $12.8044(1.7,23.9)$ & $12.1487(-26.8,51)$ & 0.907 \\
Platelet count $\left(10^{9} / \mathrm{l}\right)$ & $154.58(57.95,251.21)$ & $171.0698(41.45,300.69)$ & 0.417 \\
TOT. BILI (umol/l) & $159.9896(-16.49,336.47)$ & $84.5649(-27.15,196.71)$ & $\mathbf{0 . 0 0 1}$ \\
DIR. BILI (umol/l) & $94.79(-7.74,197.32)$ & $69.0938(-24.37,130.55)$ & $\mathbf{0 . 0 0 4}$ \\
Total protein $(\mathrm{g} / \mathrm{l})$ & $67.582(52.79,82.37)$ & $29.288(21.41,37.17)$ & 0.404 \\
Albumin $(\mathrm{g} / \mathrm{l})$ & $25.186(17.7,32.68)$ & $64.684(21.07,108.29)$ & $\mathbf{0 . 0 0 2}$ \\
ALT $(\mathrm{U} / \mathrm{l})$ & $65.054(-1.74,131.84)$ & $125.727(14.74,236.72)$ & 0.965 \\
AST $(\mathrm{U} / \mathrm{l})$ & $103.5(23.08,183.92)$ & $457.904(20.18,895.62)$ & $270.728(7.79,533.67)$ \\
ALP $(\mathrm{U} / \mathrm{l})$ & $287.149(77.53,496.77)$ & $2.23(1.45,3.01)$ & $\mathbf{0 . 0 1 6}$ \\
GGT $(\mathrm{U} / \mathrm{l})$ & $248.205(-32.79,529.21)$ & $2.48(1.81,3.15)$ & 0.639 \\
INR & $2.942(1.65,4.23)$ & $22.11(15.94,28.26)$ \\
CPS & $2.74(2.18,3.31)$ & $133.704(128.03,139.37)$ \\
MELDNa & $33.9(26.71,41.09)$ & $3.9506(3.35,4.650)$ & $\mathbf{0 . 0 0 1}$ \\
Sodium (mmol/l) & $130.214(121.99,138.43)$ & $73.6643(48.76,98.64)$ & $\mathbf{0 . 0 1 7}$ \\
Potassium (mmol/l) & $4.331(3.23,5.43)$ & $5.4277(2.24,8.62)$ & $\mathbf{0 . 0 0 1}$ \\
Creatinine (umol/l) & $271.3548(127.06,415.74)$ & $\mathbf{0 . 0 0 1}$ \\
BUN (umol/l) & $17.6812(5.1,30.3)$ & $\mathbf{0 . 0 0 5}$
\end{tabular}

Abbreviations: ALT: alanine aminotransferase; ALP: alkaline phosphatase; GGT: gamma glutamyl transferase; AST: aspartate aminotransferase; BUN: blood urea nitrogen; WBC: white blood cell; CPS: Child-Pugh score; MELDNa: model for end-stage liver disease sodium; Cr: Creatinine; T. Bil.: total bilirubin; D. Bil.: direct bilirubin; ${ }^{*} p<0.05$ : statistically significant.

conjugated), protein, albumin, alanine aminotransferase (ALT), and aspartate aminotransferase (AST). Serum sodium, potassium, blood urea nitrogen (BUN), and creatinine were also done for all patients. All patients were tested for HBsAg and anti-HCV antibodies to determine the cause of liver cirrhosis. The Child-Pugh scoring (CPS) system was used for assessing the severity of liver disease on patient presentation (26). The scoring system takes into account the serum albumin, serum prothrombin time or international normalized ratio (INR), and bilirubin as well as the presence of fluid retention and encephalopathy; each of which is given a numerical score. There are 3 grades: A, B, and C depending on the total scores. Model for end-stage liver disease sodium (MELD-Na) was also calculated for all patients [24]. Urine analysis (proteins, leucocytes, erythrocytes, pus cell, and other urine abnormalities) was done for all patients. Those with a positive leucocyte or nitrite on urinalysis had a follow-up urine culture.

2.3.2. Ascitic Fluid Analysis. Ascitic fluid analysis including cell count and differentials, albumin, and protein was done for all patients. Ascitic fluid culture: about 5-10 mls of ascitic fluid were collected during the diagnostic abdominal paracentesis and put into sterile culture bottle at the patient bed side. Ascitic fluid culture was done by an experienced biomedical scientist by inoculating the ascitic fluid onto the blood agar and MacConckey agar. The preliminary results were obtained after 48 hours, followed by conventional biochemical identification tests. The diagnosis of spontaneous bacterial peritonitis in patients with cirrhosis was based on the positive culture with no more than 1 species of organism and/or neutrophil cell counts in the ascitic fluid $\left(>250 / \mathrm{mm}^{3}\right)$ regardless of the result of the ascitic fluid culture.

2.3.3. Imaging Studies. Abdominal ultrasound scan was conducted for all patients, and the following information was recorded: liver and spleen size, changes on the liver surface and edges, presence of ascites, kidney size, and presence of liver mass. In patients with clinical diagnosis of pneumonia, chest X-ray was done for all of them, and areas of consolidation were noted.

2.3.4. Ethical Approval and Informed Consent. This study was conducted in accordance with the Helsinki Declaration on Human Experimentation, Sixth Revision (October 2008) and approved by the Institutional Ethical and Review Committee of the St. Dominic Hospital. The study was fully explained to the patients, and those who agreed to be part were asked to sign an informed consent. For patients who were drowsy, confused, and comatose on account of $\mathrm{HE}$, written consent was obtained from caregivers.

2.3.5. Statistical Analysis. Statistical Package for Social Sciences (IBM SPSS, version 23) statistical software was used to analyze the data. Descriptive statistics were undertaken for all variables and data presented in appropriate tables. A subgroup analysis was done to compare sociodemographic characteristics and clinical features of patients with AKI with and without ACLF. Etiology of cirrhosis of the liver and the prevalence of AKI were determined. Associations between $\mathrm{AKI}$ and the clinical or laboratory parameters using Chisquare were also determined. A multivariable regression analysis was conducted to determine if any of the clinical, 
TABLE 8: Multivariable regression analysis of independent variables associated with AKI.

\begin{tabular}{lccccccc}
\hline Variables & $B$ & S.E. & Wald & $p$ value & $\begin{array}{c}\text { Odds } \\
\text { ratio }\end{array}$ & \multicolumn{2}{c}{$95 \%$} \\
\hline TOT. & -0.026 & 0.015 & 2.941 & 0.086 & 0.974 & 0.945 & 1.004 \\
BILI & & & & & & & \\
DIR. BILI & 0.031 & 0.022 & 1.949 & 0.163 & 1.032 & 0.987 & 1.078 \\
Albumin & 0.059 & 0.059 & 0.973 & 0.324 & 0.943 & 0.839 & 1.060 \\
ALP & -0.004 & 0.002 & 4.321 & $\mathbf{0 . 0 3 8}$ & 0.996 & 0.993 & 1.000 \\
INR & -1.160 & 0.567 & 4.192 & $\mathbf{0 . 0 4 1}$ & 0.314 & 0.103 & 0.952 \\
CPS & -0.462 & 0.240 & 3.696 & 0.055 & 0.630 & 0.393 & 1.009 \\
MELDNA & 0.595 & 0.134 & 19.755 & $<\mathbf{0 . 0 0 1}$ & 1.813 & 1.013 & 2.357 \\
Sodium & 0.142 & 0.066 & 4.683 & $\mathbf{0 . 0 3 0}$ & 1.153 & 1.019 & 1.311 \\
Potassium & -0.366 & 0.512 & 0.510 & 0.475 & 0.694 & 0.254 & 1.893 \\
BUN & 0.210 & 0.080 & 6.902 & $\mathbf{0 . 0 0 9}$ & 1.234 & 1.055 & 1.443 \\
Infection & 1.524 & 0.866 & 3.097 & 0.078 & 4.589 & 0.841 & 2.043 \\
ENCEP & -0.481 & 0.809 & 0.353 & 0.552 & 0.618 & 0.127 & 3.020 \\
Constant & -28.238 & 10.383 & 7.396 & 0.007 & 0.000 & & \\
\hline
\end{tabular}

Abbreviations: ALP: alkaline phosphatase; BUN: blood urea nitrogen; CPS: Child-Pugh score; MELDNa: model for end-stage liver disease sodium; T. Bil.: total bilirubin; D. Bil.: direct bilirubin; ENCEP: encephalopathy; ${ }^{*} p<$ 0.05 : statistically significant.

laboratory parameters and prognostic scores (CPS, MELDNa) were a predictor of AKI. $p$ value $<0.05$ was considered statistically significant in all analysis.

\section{Results}

3.1. Demographic Characteristics and Clinical Features of Cirrhotic Patients with and without AKI. A total of 179 patients were recruited, comprising of 117 (65.4\%) males. Their ages ranged from 20 to 77 years with a mean age of 49.94 and 45.84 years for those with and without AKI, respectively. The prevalence of AKI was $27.9 \%$ (50/179). Out of 50 participants with AKI, $64.0 \%$ (32/50) died, contributing to $41.0 \%$ of all inpatient mortality amongst participants. There was a strong association between AKI and death $(p 0.001)$. The clinical features significantly associated with AKI were infection and encephalopathy. There was no difference in terms of duration of stay between patients admitted with and without AKI ( $p 0.578)$. Other demographics are as shown in Table 1.

3.2. Demographic Characteristics of Cirrhotic Patients with $A K I$ with and without ACLF. In subgroup analyses, out of the 50 patients with AKI, ACLF was diagnosed in 16 patients with infection as the main precipitating factor. All the patients diagnosed with ACLF with AKI died compared with $47.1 \%$ of those with AKI without ACLF which was statistically significant ( $p 0.001)$ (Table 2$)$.

3.3. Child-Pugh Classification. The majority of patients admitted had CPS class C, 64.8\% (116/179), and class B, 26.3\% (47/179) (Table 3).

3.4. Causes of Liver Cirrhosis. The major causes of liver cirrhosis were significant alcohol consumption and hepatitis
B virus in 72 (40.2\%) and 65 (36.3\%) of participants, respectively. The other causes found were hepatitis $\mathrm{C}$ virus in 6 (3.4\%), nonalcoholic fatty liver disease (NAFLD) in 5 $(2.8 \%)$, and schistosomiasis in $4(2.2 \%)$. In $13(7.3 \%)$ of the patients, the causes of their liver cirrhosis were not identified, and other causes are as shown in Table 4.

3.5. Precipitating Factors for All AKI. The major precipitating factors of AKI were infections (30/50, 60.0\%), hypovolemia $(10 / 50,20.0 \%)$ resulting from gastrointestinal bleeding $(5 / 50,10 \%)$ and gastroenteritis $(5 / 50,10 \%)$, and refractory ascites $(8 / 50,16.0 \%)$. Unknown precipitants accounted for $4.0 \%(2 / 50)$ (Table 5$)$.

3.6. AKI Stage and Its Effect on In-Hospital Mortality. Out of the 50 patients diagnosed with AKI, the majority of them had AKI stages 2 and 3. Patients who died had 6 times the odds of having Stage 1b AKI (compared to those who had Stage 1a AKI though this was not statistically significant [ $\operatorname{COR}(95 \% \mathrm{CI})=6(0.42-85.2)]$. Stage 2 and 3 AKI was associated with increased odds (statistically significant) of mortality compared to stage 1a with odds ratios of 10.29 (1.02-103.95) and 48 (3.64-631.76), respectively (Table 6).

3.7. Laboratory Parameters of the Study Participants. The laboratory parameters associated with AKI were high bilirubin, mainly conjugated bilirubin, low serum albumin, high international normalize ratio (INR), low sodium and potassium, high creatinine and BUN, alkaline phosphatase (ALP), CPS, and MELDNa (Table 7).

3.8. Multivariable Regression Analysis of Independent Variables Associated with AKI. ALP, INR, MELDNa, sodium, and BUN were independent predictors of AKI (Table 8).

\section{Discussion}

$\mathrm{AKI}$ is one of the most severe and life-threatening complications of cirrhosis with an estimated mortality of about $50 \%$ in a month and $65 \%$ within a year [8]. Infection and hypovolemia mainly due to upper and lower gastrointestinal bleeding and over diuresis have been found to be the main precipitating factors of AKI in liver cirrhosis [16]. This study is aimed at determining the prevalence, precipitating factors, predictors, and in-hospital mortality of AKI in patients with liver cirrhosis admitted in a hospital in Ghana. This study represents the first ever report on AKI in patients with liver cirrhosis to the best of our knowledge in Ghana. The prevalence of AKI in this study was $27.9 \%$, which is in agreement with the published literature $[4,5,7]$. A similar prevalence of $28.0 \%$ was reported by Gameiro et al. [26], in their study in Portugal. A lower prevalence of $12.9 \%$ and $17.0 \%$ were reported by Choi et al. [3] and Terra et al. [27], respectively, in their studies. Wu et al. [28] and Hampel et al. [29] also reported $23 \%$ and $25 \%$ of AKI in their patients, respectively, which were slightly lower than the prevalence obtained in this study. Lasheen et al. [2] and Lins et al. [30]reported a prevalence of $43.8 \%$ and $53.9 \%$ in their studies, respectively, which were higher than $27.9 \%$ in this current study. The 
difference maybe is a result of the demographic characteristics of the patients as well as the severity and etiology of liver disease, risk factors for AKI, and variations of several thresholds for serum creatinine used to define AKI.

Acute kidney failure can be triggered by events such as overdose of diuretics, gastrointestinal bleeding, largevolume paracentesis without albumin replacement, and bacterial infections [31]. The major precipitating factors in this study were infections $(60.0 \%)$, hypovolemia due to UGIB and gastroenteritis (20.0\%), and refractory ascites (16.0\%). This is similar to the precipitating factors reported by Lasheen et al. [2] and Gomes et al. [32] in their study. They documented infection and hypovolemia as major precipitating factors of AKI. Belcher et al. [33] also published hypovolemia and infections as major precipitating factors. Triggers of AKI should be identified early and dealt on to improve the outcome of these patients.

The estimated mortality of patients with AKI in patients with liver cirrhosis is around $50 \%$ in a month and $65 \%$ within a year [8]. Belcher et al. [33] in a prospective multicentric study involving 192 cirrhotic patients reported $26 \%$ intrahospital mortality, while Scott et al. [34] reported intrahospital mortality of $31.8 \%$ in their study. Gomes et al. [32] and Allegretti et al. [35] documented mortality rates of $45 \%$ and $46 \%$ in their studies, respectively, within 3 months of AKI diagnosis. Wong et al. [36] also observed a 34\% mortality rate in 30 days. Results of meta-analysis demonstrated that the overall mortality of renal dysfunction among patients with liver cirrhosis and UGIB was $46 \%$, with significant heterogeneity [37]. In the current study, the mortality rate among patients with AKI was as high as $64.0 \%$. However, in those admitted with AKI and concomitant ACLF, the mortality rate was $100 \%$. This confirms the high shortterm mortality associated with ACLF (22). Stages of AKI significantly influenced the in-hospital mortality in this study. Patients with more severe stages of AKI, i.e., stages 2 and 3 , had the higher odds of mortality compared to those with stages $1 \mathrm{~A}$. This is similar to a study conducted by $\mathrm{Xu}$ et al. [21]. The variations among mortality rates maybe as a result of differences in the study populations, the underlying precipitating factors of AKI, etiology and severity of liver disease, stage of AKI, and resources available for managing the patients. Vasoactive drugs such as terlipressin have been found to improve the outcome of patients with liver cirrhosis and AKI, especially those precipitated by gastrointestinal bleeding [21]. None of the patients in this study was administered with terlipressin or any other vasoactive drugs because of nonavailability at the study site. Terlipressin and other vasoactive drugs are also not widely available in Ghana, are expensive, not covered by Ghana's national health insurance, and therefore are not affordable to the majority of patients. Patients with AKI in this study were managed by treating the precipitating factors and managing the underlying causes of liver cirrhosis.

Patients who developed AKI in this study had laboratory values suggesting more advanced liver disease: higher bilirubin and INR and lower albumin and sodium values. At the same time, CPS and MELDNa were higher in patients with AKI compared to those without. Patients with AKI had infection and encephalopathy more than those without. However, high BUN, high MELDNa, high INR, and ALP were clinical parameters and scores that were independent predictors of AKI. Tariq et al. [38] reported MELD, CPS stage C, presence of ascites, and presence of sepsis/septic shock as predictors associated with AKI. Gameiro et al. [39] in their study also identified MELDNa and neutrophil-to-lymphocyte ratio as an independent predictive factor of AKI. Other studies have also reported CPS, INR, total bilirubin, serum albumin, platelet count, MELD, total leucocyte count, SBP, and shock as risk factors for AKI [40]. Patients with advanced liver cirrhosis who develop infections should be screened early for AKI and treatment instituted to reduce mortality.

\section{Conclusion}

AKI was common among patients with liver cirrhosis admitted at SDH with high in-patient mortality especially AKI associated with ACLF. The major precipitating factors of AKI were infection, hypovolemia from gastrointestinal bleeding and gastroenteritis, and refractory ascites. ALP, INR, MELDNa, serum sodium, and BUN were independent predictors of AKI. Strategies to prevent and treat precipitating factors early may lead to an improvement in the outcome of these patients. A similar study should be done on a larger scale country-wide in multiple centres and regions to get a well-balanced prevalence of AKI. In addition, a study should be conducted to determine the prevalence of multidrug-resistant bacteria in these patients, which is increasing worldwide.

\section{Data Availability}

The data used to support the findings of this study are available from the corresponding author upon request.

\section{Conflicts of Interest}

The authors declare that they have no conflicts of interest.

\section{Acknowledgments}

The authors are grateful to all patients who participated in this study.

\section{References}

[1] A. Duah and K. N. Nkrumah, "Prevalence and predictors of spontaneous bacterial peritonitis in cirrhotic patients with ascites admitted at a medical block in Korle-Bu Teaching Hospital, Ghana," The Pan African Medical Journal, vol. 33, p. 35, 2019.

[2] N. M. Lasheen, A. A. Elsawy, N. M. Nor Eldin, and K. M. Okasha, "Acute kidney injury in patients with liver cirrhosis," Tanta Medical Journal, vol. 45, no. 4, pp. 192-197, 2017.

[3] Y. J. Choi, J. H. Kim, J. K. Koo et al., "Prevalence of renal dysfunction in patients with cirrhosis according to ADQI-IAC working party proposal," Clinical and Molecular Hepatology, vol. 20, no. 2, pp. 185-191, 2014. 
[4] G. Garcia-Tsao, C. R. Parikh, and A. Viola, "Acute kidney injury in cirrhosis," Hepatology, vol. 48, no. 6, pp. 20642077, 2008.

[5] P. Tandon, M. T. James, J. G. Abraldes, C. J. Karvellas, F. Ye, and N. Pannu, "Relevance of new definitions to the incidence and prognosis of acute kidney injury in hospitalized patients with cirrhosis: a retrospective population-based cohort study," PLoS One, vol. 11, no. 8, article e0160394, 2016.

[6] A. Davenport, M. F. Sheikh, E. Lamb, B. Agarwal, and R. Jalan, "Acute kidney injury in acute-on-chronic liver failure: where does hepatorenal syndrome fit?," Kidney International, vol. 92, no. 5, pp. 1058-1070, 2017.

[7] A. Cardenas, P. Ginès, J. Uriz et al., "Renal failure after upper gastrointestinal bleeding in cirrhosis: incidence, clinical course, predictive factors, and short-term prognosis," Hepatology, vol. 34, no. 4, pp. 671-676, 2001.

[8] G. Fede, G. D’Amico, V. Arvaniti et al., "Renal failure and cirrhosis: a systematic review of mortality and prognosis," Journal of Hepatology, vol. 56, no. 4, pp. 810-818, 2012.

[9] S. G. Coca, B. Yusuf, M. G. Shlipak, A. X. Garg, and C. R. Parikh, "Long-term risk of mortality and other adverse outcomes after acute kidney injury: a systematic review and meta-analysis," American Journal of Kidney Diseases, vol. 53, no. 6, pp. 961-973, 2009.

[10] F. Salerno, A. Gerbes, P. Gines, F. Wong, and V. Arroyo, "Diagnosis, prevention, and treatment of hepatorenal syndrome in cirrhosis," Gut, vol. 56, no. 9, pp. 1310-1318, 2007.

[11] S. Dasarathy, "Consilience in sarcopenia of cirrhosis," Journal of Cachexia, Sarcopenia and Muscle, vol. 3, no. 4, pp. 225-237, 2012.

[12] P. Angeli, P. Ginès, F. Wong et al., "Diagnosis and management of acute kidney injury in patients with cirrhosis: revised consensus recommendations of the International Club of Ascites," Journal of Hepatology, vol. 62, no. 4, pp. 968-974, 2015.

[13] A. Khwaja, "KDIGO clinical practice guidelines for acute kidney injury," Nephron. Clinical Practice, vol. 120, no. 4, pp. c179-c184, 2012.

[14] A. Kellum, U. Mark, and M. L. Unruh, "Acute kidney injury," BMJ Clinical Evidence, vol. 2011, 2011.

[15] P. Ginès and R. Schrier, "Renal failure in cirrhosis," The New England Journal of Medicine, vol. 361, no. 13, pp. 1279-1290, 2009.

[16] S. Fasolato, P. Angeli, L. Dallagnese et al., "Renal failure and bacterial infections in patients with cirrhosis: epidemiology and clinical features," Hepatology, vol. 45, no. 1, pp. 223-229, 2007.

[17] D. Du Cheyron, B. Bouchet, J. Parienti, M. Ramakers, and P. Charbonneau, "The attributable mortality of acute renal failure in critically ill patients with liver cirrhosis," Intensive Care Medicine, vol. 31, no. 12, pp. 1693-1699, 2005.

[18] R. L. Mehta, J. Cerdá, E. Burdmann et al., "International Society of Nephrology's 0 by 25 initiative for acute kidney injury (zero preventable deaths by 2025): a human rights case for nephrology," Lancet, vol. 385, no. 9987, pp. 2616-2643, 2015.

[19] J. I. Farooqi, H. Ahmed, Q. Ikramullah, F. Ahmad, and M. Rehman, "Predictors of esophageal varices in patients with liver cirrhosis," Journal of Postgraduate Medical Institute, vol. 21, no. 1, pp. 60-64, 2007.

[20] The European Association for the Study of the Liver, "EASL clinical practice guidelines for the management of patients with decompensated cirrhosis," Journal of Hepatology, vol. 69, no. 2, pp. 406-460, 2018.

[21] X. Xu, B. Liu, S. Lin et al., "Terlipressin may decrease inhospital mortality of cirrhotic patients with acute gastrointestinal bleeding and renal dysfunction: a retrospective multicenter observational study," Advances in Therapy, vol. 37, no. 10, pp. 4396-4413, 2020.

[22] R. Moreau, R. Jalan, P. Gines et al., "Acute-on-chronic liver failure is a distinct syndrome that develops in patients with acute decompensation of cirrhosis," Gastroenterology, vol. 144, no. 7, pp. 1426-1437.e9, 2013.

[23] K. P. Moore, F. Wong, P. Gines et al., "The management of ascites in cirrhosis: report on the consensus conference of the international ascites club," Hepatology, vol. 38, no. 1 , pp. 258-266, 2003.

[24] A. T. Blei and J. Cordoba, "Hepatic encephalopathy," The American Journal of Gastroenterology, vol. 96, no. 7, pp. 1968-1976, 2001.

[25] A. K. Mahassadi, F. Y. Bathaix, C. Assi et al., "Usefulness of noninvasive predictors of oesophageal varices in black African cirrhotic patients in Cote d'Ivoire (West Africa)," Gastroenterology Research and Practice, vol. 2012, Article ID 216390, 10 pages, 2012.

[26] J. Gameiro, J. A. Fonseca, J. M. Dias et al., "Prediction of acute kidney injury in cirrhotic patients: a new score combining renal, liver, and inflammatory markers," International Journal of Nephrology and Renovascular Disease, vol. 11, pp. 149-154, 2018.

[27] C. Terra, M. Guevara, A. Torre et al., "Renal failure in patients with cirrhosis and sepsis unrelated to spontaneous bacterial peritonitis: value of MELD score," Gastroenterology, vol. 129, no. 6, pp. 1944-1953, 2005.

[28] C. C. Wu, L. K. Yeung, W. S. Tsai et al., "Incidence and factors predictive of acute renal failure in patients with advanced liver cirrhosis," Clinical Nephrology, vol. 65, no. 1, pp. 28-33, 2006.

[29] H. Hampel, G. D. Bynum, E. Zamora, and H. B. El-Serag, "Risk factors for the development of renal dysfunction in hospitalized patients with cirrhosis," The American Journal of Gastroenterology, vol. 96, no. 7, pp. 2206-2210, 2001.

[30] P. R. Gessolo Lins, W. S. Carvalho Padilha, C. F. Magalhaes Giradin Pimentel, M. Costa Batista, and A. F. Teixeira de Gois, "Risk factors, mortality, and acute kidney injury outcomes in cirrhotic patients in the emergency department," $B M C$ Nephrology, vol. 19, no. 1, pp. 1-8, 2018.

[31] A. L. Gerbes, "Liver cirrhosis and kidney," Digestive Diseases, vol. 34, no. 4, pp. 387-390, 2016.

[32] C. G. Gomes, M. V. Andrade, L. Resende Guedes et al., "Clinical aspects and prognosis evaluation of cirrhotic patients hospitalized with acute kidney injury," Canadian Journal of Gastroenterology \& Hepatology, vol. 2019, article 6567850, 2019.

[33] J. M. Belcher, G. Garcia-Tsao, A. J. Sanyal et al., "Association of AKI with mortality and complications in hospitalized patients with cirrhosis," Hepatology, vol. 57, no. 2, pp. 753762, 2013

[34] R. A. Scott, A. S. Austin, N. V. Kolhe, C. W. McIntyre, and N. M. Selby, "Acute kidney injury is independently associated with death in patients with cirrhosis," Frontline Gastroenterology, vol. 4, no. 3, pp. 191-197, 2013.

[35] A. S. Allegretti, G. Ortiz, J. Wenger et al., "Prognosis of acute kidney injury and hepatorenal syndrome in patients with 
cirrhosis: a prospective cohort study," International Journal of Nephrology, vol. 2015, Article ID 108139, 2015.

[36] F. Wong, J. G. O'Leary, K. R. Reddy et al., "New consensus definition of acute kidney injury accurately predicts 30-day mortality in patients with cirrhosis and infection," Gastroenterology, vol. 145, no. 6, pp. 1280.e1-1288.e1, 2013.

[37] Z. Bai, M. Primignani, X. Guo, K. Zheng, H. Li, and X. Qi, "Incidence and mortality of renal dysfunction in cirrhotic patients with acute gastrointestinal bleeding: a systematic review and meta-analysis," Expert Review of Gastroenterology \& Hepatology, vol. 13, no. 12, pp. 1181-1188, 2019.

[38] R. Tariq, Y. Hadi, K. Chahal, S. Reddy, H. Salameh, and A. K. Singal, "Incidence, mortality and predictors of acute kidney injury in patients with cirrhosis: a systematic review and meta-analysis," Journal of Clinical and Translational Hepatology, vol. 8, no. 2, pp. 135-142, 2020.

[39] J. C. P. Puentes, H. Rocha, S. Nicolau, and G. Ferrao, "Effectiveness of the MELD/Na score and Child-Pugh score for the identification of palliative care needs in patients with cirrhosis of the liver," Indian Journal of Palliative Care, vol. 24, no. 4, pp. 526-528, 2018.

[40] M. S. Arora, R. Kaushik, S. Ahmad, and R. M. Kaushik, "Profile of acute kidney injury in patients with decompensated cirrhosis at a tertiary-care center in Uttarakhand, India," Digestive Diseases, vol. 38, no. 4, pp. 335-343, 2020. 